\title{
Activité anti-toxoplasmose, screening phytochimique et étude de la cytotoxicité de l'extrait éthanolique 70\% de Hunteria eburnea Pichon (Apocynaceae)
}

\section{Camara Djeneb}

Laboratoire de Botanique, Unité de Formation et de Recherche Biosciences, Abidjan, Université Félix Houphouët-Boigny, Cote d'Ivoire

\section{Coulibaly Kiyinlma}

UFR Sciences biologiques, Département de production Végétale. Université Peleforo Gon Coulibaly, Korhogo, Côte d'Ivoire

\section{Kanga Yao}

Laboratoire de Botanique, Unité de Formation et de Recherche Biosciences, Abidjan, Université Félix Houphouët-Boigny, Cote d'Ivoire

\section{Bisanz Cordelia}

\section{Delauw Marie-France}

Laboratoire Timc-Imag de l'Université Grenoble-Alpes, France

\section{Zirihi Guédé Noël}

Laboratoire de Botanique, Unité de Formation et de Recherche Biosciences, Abidjan, Université Félix Houphouët-Boigny, Cote d'Ivoire

\section{Doi: 10.19044/esj.2018.v14n30p37 URL:http://dx.doi.org/10.19044/esj.2018.v14n30p37}

\begin{abstract}
Hunteria eburnea is a medicinal plant used in traditional medicine in the Sassandra Region (Ivory Coast) in the treatment of malaria and skin diseases. The aim of this study is to study the inhibitory effect of $70 \%$ ethanolic extract of Hunteria eburneaa stem bark on Toxoplasma gondii, a protozoan parasite such as Plasmodium falciparum that causes toxoplasmosis. The $70 \%$ ethanolic extract was obtained from the parts of the plant that are used by traditional health practitioners in the Haut-Sassandra Region (Ivory Coast).The $70 \%$ ethanolic extract of Hunteria eburnea stem bark revealed high anti-Toxoplasma gondii activity with an estimated $\mathrm{IC}_{50}$ of $0.72 \mathrm{mg} / \mathrm{mL}$ and no cytotoxicity to HFF cells. (Human Foreskin Fibroblasts). Also, the phytochemical screening of this extract revealed the presence of sterols / triterpenes as well as alkaloids. This result indicates a promising source of new anti-Toxoplasma drugs from Hunteria eburnea, a West African medicinal plant.
\end{abstract}


Keywords: HFF cells, Ivory Coast, Medicinal plants, Toxoplasma gondii

\section{Résumé}

Hunteria eburnea est une plante médicinale utilisée en médecine traditionnelle dans la Région du Haut-Sassandra (Côte d'Ivoire) dans le traitement du paludisme et des maladies de la peau. Le but de cette étude, est d'étudier l'effet inhibiteur de l'extrait éthanolique $70 \%$ de l'écorce de tige de Hunteria eburneaa sur Toxoplasma gondii, un parasite protozoaire comme Plasmodium falciparum responsable de la toxoplasmose. L'extrait éthanolique $70 \%$ a été obtenu à partir des parties de la plante qui sont utilisées par les tradipraticiens de santé de la Région du Haut-Sassandra (Côte d'Ivoire). L'extrait éthanolique $70 \%$ de l'écorce de tige de Hunteria eburneaa révélé une activité anti-Toxoplasma gondii élevée avec une $\left(\mathrm{CI}_{50}\right)$ estimé à $0,72 \mathrm{mg} / \mathrm{mL}$ et une absence de cytotoxicité vis-à-vis des cellules HFF (Human Foreskin Fibroblasts). Aussi, le criblage phytochimique de cet extrait a mis en évidence la présence de stérols/triterpènes ainsi que des alcaloïdes. Ce résultat indique donc une source prometteuse de nouveaux médicaments antiToxoplasma à partir de Hunteria eburnea, une plante médicinale ouest africaine.

Mots clés: Cellules HFF, Côte d'Ivoire, Plantes médicinales, Toxoplasma gondii

\section{Introduction}

Toxoplasma gondii est un parasite cosmopolite. Des études épidémiologiques chez l'homme ont montré sa large distribution géographique et sa forte prévalence. L'incidence de la toxoplasmose dans la population générale est difficile à évaluer car l'infection est le plus souvent asymptomatique. La toxoplasmose affecte environ 7 à $80 \%$ de la population mondiale mais le pourcentage de personnes séropositives pour l'infection toxoplasmique varie d'un pays à l'autre en fonction des groupes ethniques, des habitudes culinaires et des conditions d'hygiène (Tenter et al., 2000). L'incidence de la toxoplasmose dans la population générale est difficile à évaluer car l'infection est le plus souvent asymptomatique et ne fait pas l'objet d'une déclaration obligatoire auprès des services de santé (Porto et al., 2008). Les traitements actuels de la toxoplasmose sont la combinaisonde 2,4diaminopyrimidines (pyriméthamine, triméthoprime) et les sulfamides dont sulfadiazine et sulfaméthoxazole (Gras et al., 2005). En raison du manque de spécificité et de l'efficacité limitée de ces traitements actuels, il y a un besoin impérieux de rechercher de nouveaux composés pour traiter la toxoplasmose. Les produits d'origine naturelle sont une source importante de découverte de nouvelles entités chimiques dont sont issus bons nombres de médicaments. 
Ainsi, que ce soit dans le domaine du cancer, des maladies neurodégénératives ou des maladies infectieuses, les substances naturelles sont sollicitées pour la découverte de nouveaux médicaments. Avant les années 1990, le principe actif d'environ $80 \%$ de médicaments vendus était isolé de produits d'origine naturelle ou des analogues inspirés de produits naturels (Mariam, 2012). Parmi eux, on peut citer des antibiotiques (la pénicilline, la tétracycline, l'érythromycine), des antiparasitaires (la quinine, l'artémisinine), des immunosuppresseurs (cyclosporine, rapamycines) et des anticancéreux (taxol, doxorubicine). Le principe actif de ces célèbres médicaments a été isolé souvent à partir des plantes, mais aussi à partir des champignons (pénicilline) ou des bactéries (Traoré, 2012). C'est pour cette raison qu'une enquête ethnobotanique a été réalisée auprès des tradipraticiens de santé. Suite à cette enquête Hunteria eburnea a été sélectionnée en raison de la fréquence de son utilisation par les tradipraticiens de santé pour traiter et guérir les personnes atteintes du paludisme. Le but de ce travail est d'évaluer in vitro l'activité de l'extrait éthanolique $70 \%$ de Hunteria eburnea, une plante médicinale de la pharmacopée ivoirienne sur Toxoplasma gondii et de déterminer sa cytotoxicité.

\section{Materiel et Methodes Matériel végétal}

Le matériel végétal utilisé est constitué des écorces de tronc de Hunteria eburnea récoltées dans le Département d'Issia dont l'identification a été faite par comparaison avec un échantillon de l'herbier du Centre National de Floristique de l'Université Felix Houphouët Boigny (Abidjan Côte d'Ivoire) sous le numéro 15904 (Forêt du Banco, 01 /06/1981, Aké-Assi L).

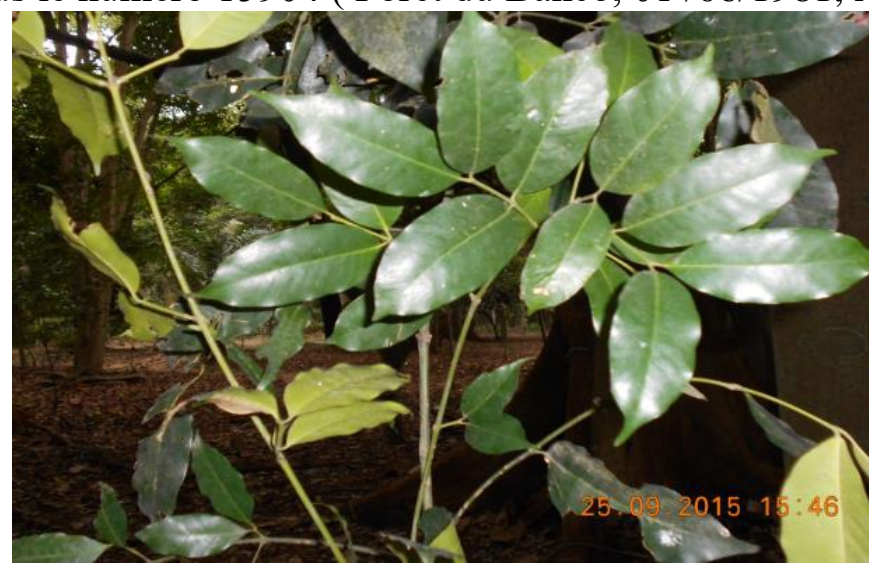

Figure 1 : Rameau feuillé de Hunteria eburnea, (Issia, 2015)

\section{Matériel biologique}

Le matériel biologique est composé de cellules HFF (Human Foreskin Fibroblasts) qui sont des cellules humaines, de fibroblastes issus du derme et 
du parasite $T$. gondii RH-YFP2 (souche RH exprimant deux gènes codant pour la yellow fluorescent protein).

\section{Préparation des extraits:}

Les écorces de tige de Hunteria eburnea récoltées, ont été découpées, rincées à l'eau et séchées à l'abri du soleil. Ces organes végétaux séchés ont été ensuite réduits en poudre fine grâce à un broyeur électrique IKA-MAG RTC. On obtient une poudre de couleur grise. Les extraits (total aqueux et éthanolique $70 \%$ ) ont été préparés selon la méthode décrite par (Zirihi et al., 2003).

\section{- Extrait total aqueux:}

Cent grammes $(100 \mathrm{~g})$ de poudre des écorces sont homogénéisés dans 1 litre d'eau distillée dans un Blender (Mixer) de marque Life's Superb (LS317) pendant trois minutes à la température ambiante. Cette opération est répétée trois fois et l'homogénat obtenu est filtré successivement sur du coton hydrophile puis sur du papier Wattman $(3 \mathrm{~mm})$. A l'aide d'une étuve réglée à $50^{\circ} \mathrm{C}$, le solvant d'extraction est éliminé. L'évaporat sec récupéré, sous forme de poudre, constitue l'extrait total aqueux (ETA).

\section{- Extrait éthanolique 70\%:}

Cinq grammes $(5 \mathrm{~g})$ de l'ETA ont été dissouts dans $100 \mathrm{ml}$ d'une solution d'éthanol $70 \%$ puis homogénéisés dans un Blender. Après décantation dans une ampoule à décanter, le surnageant recueilli est filtré sur du coton pour le débarrasser de tout résidu et séché à l'étuve $\left(50^{\circ} \mathrm{C}\right)$. La poudre obtenue constitue l'extrait éthanolique 70\% (EE70\%).

\section{Criblage phytochimique}

La mise en évidence des groupes phytochimiques tels que les alcaloïdes, les stérole/triterpènes, les tannins et les saponosides a été effectuée par des méthodes qualitatives de coloration selon Harbone (1998).

\section{Test de prolifération des souches de $T$. gondii}

Les souches de $T$. gondii RH-YFP2 (souche RH exprimant deux gènes codant pour la yellow fluorescent protein), ont été maintenues en culture par passages sur tapis de cellules HFF confluents dans du milieu D10. Les parasites sont déposés sur des lamelles de verre recouvertes d'un tapis de cellules HFF confluentes. Après 30 secondes de centrifugation à environ 100 g pour accélérer la sédimentation des parasites sur les cellules HFF, les plaques de culture sont incubées à $37^{\circ} \mathrm{C}$ pendant $15 \mathrm{~min}$. Le tapis cellulaire est ensuite lavé 3 fois avec du milieu PBS (phosphate buffer saline : $\mathrm{NaCl} 137 \mathrm{mM} ; \mathrm{KCl}$ 2,7 mM ; Na2HPO4 10 mM ; KH2PO4 1,8 mM) afin d'éliminer les parasites extracellulaires. Le noyau des cellules HFF est coloré à 1'Hoechst 33258, ils auront une couleur bleu. Pour le traitement intracellulaire, on réalise une 
invasion synchronisée avec environ 100 parasites par lamelle de verre recouvert d'un tapis de cellules HFF confluentes. On remplace le milieu de culture par un milieu D10 supplémenté des extraits de plantes $(0-1 \mathrm{mg} / \mathrm{ml})$. Après $24 \mathrm{~h}$ de culture, les cellules parasitées sont fixées. Le nombre de parasites à l'intérieur des vacuoles parasitophores est comptabilisé. Les parasites qui expriment la YFP sont visualisés directement au microscope à épifluorescence (Derouin et al., 1989).

\section{Test de cytotoxicité sur les cellules HFF}

L'étude de la toxicité est inspirée de la méthode de Mossman (1983). Pour mesurer la toxicité de l'extrait éthanolique, les cellules HFF ont été ensemencées dans des plaques de 96 puits (CellStar) à raison de 3000 à 5000 cellules par puits dans $100 \mu 1$ de milieu D10. Ces cellules sont maintenues en culture pendant 24 heures (cellules en division) ou 96 heures (cellules confluentes). Par la suite elles ont été exposées pendant 24 heures à différentes concentration $(0-1000 \mu \mathrm{g} / \mathrm{ml})$ en extrait de plante solubilisé dans du tampon PBS. Cela a été fait en triplicate. La viabilité a été déterminée à l'aide du bromure de 3-(4,5-diméthylthiazol-2-yl)-2,5-diphenyl tetrazolium (MTT). L'anneau de tétrazolium qu'il contient est réduit en formazan par la succinate déshydrogénase mitochondriale des cellules métaboliquement actives, qui précipite et donne une couleur violette. La quantité du précipité formé est proportionnelle au nombre de cellules vivantes. Dans chaque puits, le MTT est ajouté à une concentration de $500 \mu \mathrm{g} / \mathrm{ml}$ et incubé pendant $3 \mathrm{~h}$ à $37^{\circ} \mathrm{C}$. Les cristaux de formazan sont solubilisés dans du diméthylsulfoxyde (DMSO) 10 mM. La mesure de la densité optique à $544 \mathrm{~nm}$ a été faite à l'aide d'un spectrophotomètre Safir (Tecan) ; cette mesure de l'absorbance permettra de déterminer la quantité relative de cellules vivantes et actives métaboliquement. Les résultats ont été exprimés en pourcentage de viabilité par rapport au contrôle sans extrait de plante. Taux viabilité $=($ Abs544 nm extrait/ Abs544 nm témoin) $\times 100$.

\section{Résultats}

\section{Activité anti-toxoplasma gondii de l'extrait éthanolique $70 \%$ de Hunteria eburnea}

Quand on observe 1'effet de 1'extrait éthanolique $70 \%$ de Hunteria eburnea sur la prolifération de $T$. gondii (figure 2), on constate qu'il inhibe la prolifération du parasite, et que cette inhibition est dose dépendante. A la concentration de $0,72 \mathrm{mg} / \mathrm{mL}$, on observe une inhibition de $50 \%$ des parasites. En effet, à la plus forte concentration en extrait de plante qui est de $1,2 \mathrm{mg} / \mathrm{mL}$, nous avons un taux d'inhibition d'environ $80 \%$. Au-delà de cette concentration la sensibilité de l'appareil est influencée par la coloration de 
l'extrait de plante. $\mathrm{La} \mathrm{CI}_{50}$ de l'extrait éthanolique $70 \%$ de Hunteria eburnea est estimée à $0,72 \mathrm{mg} / \mathrm{mL}$.

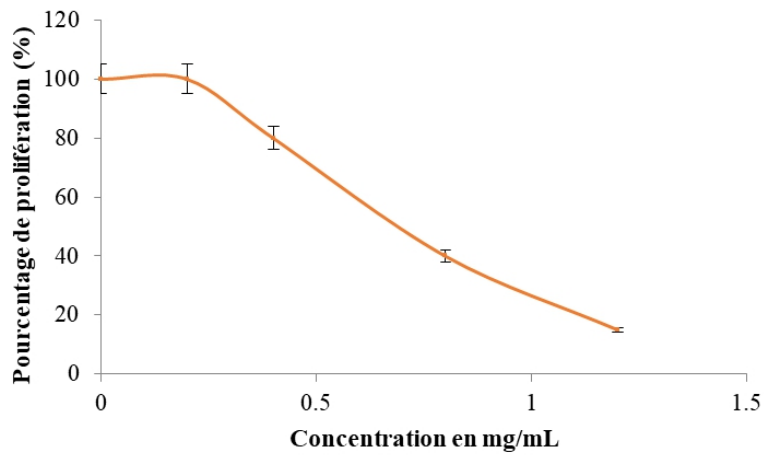

Figure 2 : Effet de l'extrait éthanolique de Hunteria eburnea sur la prolifération de $T$. Gondii l'intérieur des fibroblastes humains (HFF).

\section{Toxicité del'extrait éthanolique $70 \%$ de Hunteria eburnea sur des cellules humaines HFF}

L'observation de l'effet des extraits éthanolique de Hunteria eburnea sur la viabilité cellulaire nous apprend que quelques soit les conditions des cellules (en arrêt ou en division), Hunteria eburnea augmente le taux de viabilité (figure 3). Ce taux est cependant plus faible pour les cellules confluentes que pour les cellules en division. Il y a sûrement dans l'extrait des composés antioxydants qui aide les cellules à améliorer la performance de leur $\mathrm{SDH}$. Les cellules confluentes sont plus âgées et dans des conditions physiologiques qui font qu'elles fonctionnent moins bien que les cellules en division. On peut conclure que Hunteria eburnea n'a pas d'effet toxique sur les cellules humaines, au contraire elle améliore le fonctionnement des cellules.

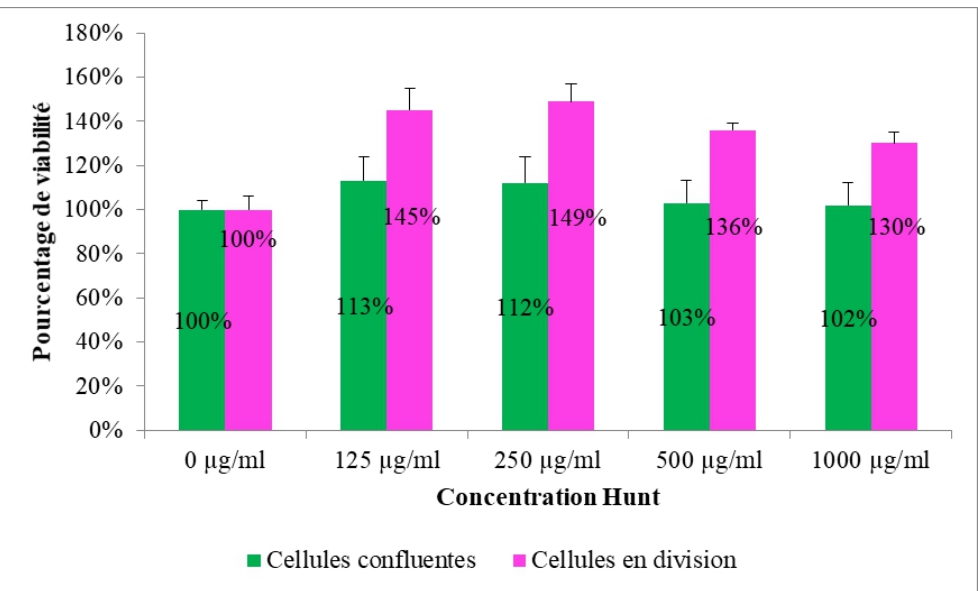

Figure 3 : Effet des différentes concentrations de l'extrait éthanolique $70 \%$ de Hunteria eburnea sur la viabilité des cellules humaines HFF. 


\section{Criblage phytochimique}

Le Tableau I donne les résultats obtenus lors du criblage phytochimique de l'extrait éthanolique de Hunteria eburnea. Les tests effectués révèlent la présence de divers métabolites secondaires dans l'extrait évalué. Dans l'extrait éthanolique $70 \%$, il a été noté la présence de stérols/triterpènes et des alcaloïdes.

Tableau I : Résultats du screening phytochimique de l'extrait éthanolique $70 \%$ de Hunteria eburnea.

\begin{tabular}{|c|c|}
\hline Molécules recherchées & Echantillon testé (EE 70 \%) \\
\hline Alcaloïdes & + \\
\hline Polyphénols & - \\
\hline Tanins galliques & - \\
\hline Tanins cathéchiques & - \\
\hline Flavonoïdes & - \\
\hline Terpènes/stérols & - \\
\hline Coumarines & - \\
\hline \multicolumn{2}{|c|}{ Saponines } \\
\multicolumn{2}{|c|}{$+:$ présence du groupe chimique } \\
$-:$ absence du groupe chimique
\end{tabular}

EE $70 \%$ : extrait éthanolique $70 \%$

\section{Discussion}

L'enquête ethnobotanique menée auprès des tradipraticiens de santé de la Région du Haut-Sassandra (Côte d'Ivoire), a révélé que l'écorce de tige de Hunteria eburnea est utilisée pour traiter les maladies parasitaires et les dermatoses. Les recettes obtenues de cette plante sont monospécifiques, ce qui constitue un avantage dans le traitement des patients. Selon N'Guessan et al. (2008) les associations de plantes mal assorties, sont parfois dangereuses. L'activité in vitrode l'extraitéthanolique $70 \%$ deHunteria eburnea effectuée sur T. gondiia a montré un taux d'inhibition d'environ $80 \%$ avec une $\mathrm{CI}_{50}$ de $0,72 \mathrm{mg} / \mathrm{mL}$. À cet égard, Hunteria eburnea pourrait être une source prometteuse de nouveaux médicaments anti-parasitaires, comme c'est le cas our plusieurs antipaludiques tels que l'artémisinine. Ce résultat pourrait en partie justifier l'utilisation courante de cette plante dans le traitement du paludisme par les tradipraticiens de santé dans la Région du Haut-Sassandra (Côte d'Ivoire). Nos résultats corroborent ceux de Benoit-Vical et al. (2000), qui ont aussi montré l'efficacité Vernonia colorata sur $T$. gondii mais avec une CI 50 de $16,3 \mathrm{mg} / \mathrm{L}$. Lorsqu'on compare cette valeur de $\mathrm{CI}_{50}$ à celle obtenue avec l'extrait aqueux de Vernonia colorata de Benoit-vical etal. (2000) ou la $\mathrm{CI}_{50}$ est de $0,0163 \mathrm{mg} / \mathrm{mL}$ on peut dire que l'activité de l'extrait éthanolique $70 \%$ de Hunteria eburneaest moindre. Cette différence de résultat pourrait s'expliquer par le fait que les protocoles expérimentaux sont très différents. Alors que nous observons directement les parasites au microscope à épifluorescence, leur étude réalise un test ELISA pour ies détecter. La 
précision de ces tests ELISA n'est pas évidente. De plus, pendant que nous incubons les parasites en présence de la drogue durant 24 heures, ces auteurs le font pendant 72 heures. La durée d'incubation ainsi que la méthode de détection du parasite peuvent avoir une influence sur les valeurs de $\mathrm{CI}_{50}$.Le résultat du test de cytotoxicité effectué sur les cellules HFF a montré que l'extrait éthanoliques de Hunteria eburnea n'est pas toxique sur les cellules humaines HFF. En effet, selon Bené (2017), lorsque le taux de viabilité d'un extrait est strictement supérieur à $30 \%$ l'extrait est déclaré non cytotoxique. Nos résultats sont conformes à ceux de Camara et al. (2016) et de Yapo et al. (2016) qui ont aussi montré que les écorces de tige de Bersama Abyssinica (Fresen.): Melianthaceae et les feuilles de Mallotus oppositifolius (Geiseler) Müller. Arg (Euphorbiaceae) utilisées en milieu traditionnel en Côte d'Ivoire n'avaient pas d'effets cytotoxiques sur les cellules HFF.

\section{Conclusion}

Ce travail a montré le potentiel thérapeutique de Hunteria eburnea sur Toxoplasma gondii .L'extrait éthanolique $70 \%$ inhibe fortement la croissance in vitro de toxoplasma gondii à des concentrations qui ne sont pas toxique sur les cultures cellulaires HFF. Aussi le résultat de la présente étude encourage les investigations futures sur cette plante dans l'optique d'isoler la ou les molécules responsables de l'efficacité anti-toxoplasmose, de manière à concevoir un médicament traditionnel amélioré (MTA) pour le traitement de la toxoplasmose.

\section{Remerciements}

Les auteurs remercient le Laboratoire Adaptation et Pathogénie des Microorganismes (LAPM) de Grenoble en France, où les études de cytotoxicité ont été réalisées, le Laboratoire de Botanique, où le séchage des plantes récoltées a été effectué et les tradipraticiens de la Région du HautSassandra.

\section{References:}

1. Bené. (2017). Plantes médicinales du Gontougo (district du Zanzan, Côte d'Ivoire) : inventaire, évaluation des activités pharmacologiques de deux plantes et formulation d'une pommade dermatologique à partir de l'extrait hydroalcoolique de Bersama abyssinica fresen. (Melianthaceae). Thèse de Unique de Botanique, Université Félix Houphouët-Boigny (Côte d'Ivoire), 200 p.

2. Benoit-Vical F., Santillana-Hayat M., Kone-Bamba D., Malli E M., \& Derouin F. (2000). Anti-toxoplasma activity of vegetal extracts used in west african traditional medicine. Parasite, $7: 3-7$. 
3. Camara D., Bené K., Gnahoue G., Fofié N.B.Y., \& Zirihi G.N. (2016). Etude ethnobotanique, évaluation de l'activité antifongique sur Candida albicans et de la toxicité sur des cellules HFF de Bersama Abyssinica (Fresen.), une plante de la pharmacopée ivoirienne. European Scientific Journal, 12 (3) : 171-1885,

4. Derouin F., \& Chastang D. (1989). In vitro effects of folate inhibitors on Toxoplasma gondii. Antim Agents and Chemoth., 33, 1753-1759.

5. Gras L., Wallon M., Pollak A., Cortina-Borja M., Evengard B, Hayde

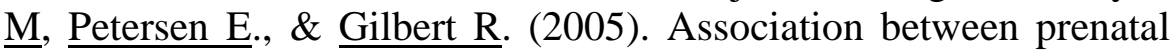
treatment and clinical manifestations of congenital toxoplasmosis in infancy: a cohort study in 13 European centres. Acta Paediatr, 94, 1721-31.

6. Harborne J B. (1998). A guide to modern techniques of plant analysis. Springer, 3rd Edn, India (New Delhi), pp 5-32.

7. Mosman T. (1983). Rapid colorimetric assay for cellular growth and survival: application to proliferation and cytotoxicity assays. Journal of Immunological Methods, 65 : 55-63.

8. N'Guessan K., Kouadio K., Kouamé N F., Traoré D., \& Aké-Assi L (2008). Etude botanique des plantes emménagogues utilisées en médecine traditionnelle par les Abbey et Krobou d'Agboville (Côted'Ivoire). Rev Med Pharm Afr, 21: 43-60.

9. Porto A. M., Amorim M. M., Coelho I.C., \& Santos LC. (2008). [Serologic profile of toxoplasmosis in pregnant women attended at a teachinghospital in Recife]. Rev Assoc Med Bras, 54, (3), 242-248.

10. Tenter AM., Heckeroth AR., \& Weiss LM. (2000). Toxoplasma gondii from animals to humans. Int J Parasitol, 30:1217-58.

11. Traoré M. (2012). Synthèses et évaluations de nouveaux composés antipaludiques et antitoxoplasmoses. Thèse Unique en Microbiologie et Parasitologie. Université de Grenoble, (France), 184p.

12. Yapo Y C V., Konkon G., Coulibaly K., Camara D., \& Zirihi G. N. (2016). Etude botanique, évaluation de l'activité antifongique sur la croissance in vitro de Candida albicans et de la toxicité sur des cellules HFF de feuilles de Mallotusoppositifolius (Geiseler) Müller. Arg (Euphorbiaceae). J. Anim. Plant Sci. 28 (1), 4330-4339.

13. Zirihi G N., Kra A M., \& Guédé-Guina F. (2003). Evaluation de l'activité antifongique de Microglossa pyrifolia (Larmarck) O. kuntze (Asteraceae) " pymi " sur la croissance in vitro de Candida albicans. Rev de Méd et de Pharma Afric, 17 : 11-19. 